\title{
Stress-only imaging: Faster, cheaper, less radiation. So what's the hold up?
}

\author{
Milena J. Henzlova, MD, Lori B. Croft, MD, and W. Lane Duvall, MD
}

\section{See related article, pp. 27-37}

Current SPECT myocardial perfusion imaging (MPI) protocols were developed several decades ago when radiation exposure, health care costs, comparative effectiveness, and down-stream testing were unheard of terms. Over the past two decades, we have witnessed significant developments in SPECT hardware and software as well as the introduction of new pharmacologic stress agents. Simultaneously, we have seen the development and maturation of alternative cardiac diagnostic technologies such as positron emission tomography (PET), coronary CT angiography (CTA), cardiac MRI, stress echocardiography, and hybrid perfusion/anatomic imaging. Thus, it is due (or perhaps overdue) to embrace new approaches to SPECT MPI.

In the current issue of the Journal, Mathur et al contribute to the current body of literature promoting "stress-only" or "stress-first" imaging. ${ }^{1}$ The authors retrospectively analyzed 1,383 consecutive patients, who underwent stress-only (or stress-first) MPI using a conventional SPECT camera equipped with Gd-153based attenuation correction technology. Like previously published literature, they find that there is a low cardiac event rate with a normal stress-only study, with an annualized cardiac event rate of $0.7 \%$. The majority of the patients $(90 \%)$ did not need rest images, as their uncorrected (42\%) and/or attenuation corrected (48\%) perfusion images were interpreted as normal. This study also adds to the current body of stress-only literature by including data on the use of attenuation correction and outcomes of abnormal stress-first studies.

The benefits of stress-only protocols are straightforward. The length of a MPI study is dramatically

From the Mount Sinai Division of Cardiology (Mount Sinai Heart), Mount Sinai Medical Center, New York, NY.

Reprint requests: W. Lane Duvall, MD, Mount Sinai Division of Cardiology (Mount Sinai Heart), Mount Sinai Medical Center, New York, NY; william.duvall@msnyuhealth.org.

J Nucl Cardiol 2013;20:17-9.

$1071-3581 / \$ 34.00$

Copyright (c) 2012 American Society of Nuclear Cardiology.

doi:10.1007/s12350-012-9652-4 decreased, the radiation exposure to patients is reduced by $30-60 \%$, and there is cost savings to the health care system. The benign prognosis of a normal stress-only study was confirmed in recently published pooled data which showed a low annual event rate at of $0.67 \%$ in $>10,000$ patients. $^{2}$ Stress-only imaging has been validated in multiple settings including inpatients, outpatients, and the emergency department, in those with $\mathrm{CAD}$ and without $\mathrm{CAD}$, and in the obese. ${ }^{3-7}$ Considering the high prevalence of normal MPI studies (60-70\%) in most large published studies, the redundancy of rest imaging is clear. Interestingly, the European MPI guidelines published in 2005 state "when the study is performed for the diagnosis of myocardial ischaemia, the stress study should be performed first because this avoids reduction of the contrast of a stress-induced defect by a previous normal study and also obviates the need for rest imaging." 8 What, then, are the issues preventing more widespread use of a more efficient, faster, less expensive, and radiation reducing protocol?

\section{WHO IS APPROPRIATE FOR STRESS-FIRST IMAGING?}

From the accumulated experience in the literature, the criteria for selecting a stress-first imaging protocol can be summarized as follows: patients with symptoms suggestive of ischemia and low to intermediate pre-test probability, an interpretable ECG, no history of documented MI, PCI, and/or CABG, a history of a recent normal functional (perhaps $<2$ years) or anatomic (normal CTA likely has a longer "warranty" period) ${ }^{9}$ study. Mathur et al add to this list patients with congestive heart failure/cardiomyopathy but it is unclear how this was defined. The authors of this article also recommend rest-stress imaging for the morbidly obese, but it would appear that this is another category suitable for stressfirst. Given other published literature demonstrating the feasibility of stress-only imaging in pre-bariatric surgery, ${ }^{7}$ it is unclear why they make this recommendation as stress-first imaging should be used to triage this subgroup of patients when one day testing is not practical due to the need for higher tracer doses. Some guidance beyond including low to intermediate pre-test risk patients and excluding those with known CAD can be found in a recent publication suggesting a simple 
score for determining eligibility for stress-first protocols. $^{10}$

Most patients and referring providers are unlikely to balk at a small risk of needing rest imaging after an unsuccessful stress-first attempt in exchange for the potential time and radiation exposure savings, either to return the following day or have a high-dose rest injection the same day. Similar to Tl-201, a stress-rest sequence could be implemented for Tc-99m MPI studies. Contrary to current US guidelines, but in accordance with the European guidelines, the second day rest imaging can be done with low tracer dose, rather than with a second high dose. ${ }^{8}$ Interestingly, this sequence was considered with the advent of Tc-99m sestamibi. ${ }^{11}$ Rather marginal difference in image quality between rest-stress and stress-rest sequence led to over 2 decades of long, radiation-rich studies. The controversy persists about the needed time between low and high Tc-99m doses. On one hand, the "shine through" phenomenon is considered to be of importance $;{ }^{12}$ on the other hand, it has been trivialized. ${ }^{13}$ Current practice varies from as low as a 1:2 to as high as a 1:4 ratio between the low and high doses. Also, the time to rest imaging after the stress dose varies considerablyfrom few minutes to several hours. It is likely that more studies or modeling are needed to clarify and codify these issues with modern SPECT cameras and software.

\section{READERS MUST BE IMMEDIATELY AVAILABLE}

Stress-first protocols come with the need for physician's presence because individual decisions need to be make prior to the test about the protocol to be used, and then stress images need to be quickly reviewed to decide on the need for rest images. This approach is labor-intensive and requires a physician to be available throughout the day. This is unlikely to be the case at many laboratories as the interpreting physician has other responsibilities during the day and only comes to read in the afternoon or evening. Economically, this makes sense in the current climate of reduced reimbursement. ${ }^{14}$ While the protocol decisions could potentially be done in advance or handled by other laboratory staff members based on triage guidelines and while current technology allows for remote viewing of MPI images, these requirements may limit stress-first imaging to high-volume hospital-based laboratories. Highvolume laboratories will also be better equipped for handling scheduling irregularities caused by a combination of stress-only, stress-rest, and rest-stress protocols.

\section{REIMBURSEMENT AND THE NEED FOR ATTENUATION CORRECTION}

The current 2012 Medicare fee schedule structure penalizes outpatient private (non-hospital owned) laboratories which represent the majority of the US laboratories for stress-only imaging. For hospital-owned laboratories, the professional reimbursement is slightly decreased (14.7\%) for stress-only studies ("single imaging") compared to two-part studies ("multiple imaging"). The technical portion is unchanged resulting in only a $1.5 \%$ reduction in global reimbursement. Private laboratories whose reimbursement is already over $1 / 3$ less than hospital laboratories see an additional $28 \%$ reduction in global reimbursement if a stress-only protocol is performed compared to a two-part study. Thus, we are faced with a strange paradox. Improved productivity and improved patient safety are not rewarded, just the opposite, they are penalized. However, there is another less visible, but real financial benefit to stress-only imaging: Laboratory throughput is improved due to the shorter test time and either more patients can be scheduled or the staffing can be reduced to maintain the same daily volumes. Another obstacle is our current inability to preapprove stress-first studies. Many insurance carriers require re-approval of "single imaging" studies when they have been pre-approved as "multiple imaging."

This article adds to the body of stress-only literature by illustrating the need for a method to correct attenuation artifacts in order for a laboratory to undertake a successful stress-first strategy. Previous study has demonstrated the ability of attenuation correction to reduce false positives, ${ }^{15,16}$ and in this reported cohort $83 \%$ of the studies felt to be abnormal without attenuation correction were reclassified as normal. In fact without the aid of attenuation correction, only $42 \%$ of the patients in the study would have been normal and the $48 \%$ of patients that were normal after attenuation correction would have had to undergo rest imaging, thereby greatly reducing the attractiveness of a stressfirst protocol. A Gd-153 scanning line source as used in this publication or CT-based attenuation correction is more likely a privilege of large laboratories due to the added hardware cost and current lack of reimbursement for its use. Prone or semi-upright imaging in addition to supine imaging can be used without additional costs with similar results to hardware-based attenuation correction, ${ }^{17}$ although it is time consuming on conventional SPECT cameras without $1 / 2$ time imaging software and therefore unattractive to many practices. Until the additional expenses associated with stress-first imaging are reimbursed, large hospital laboratories are likely to be the leaders in this new paradigm.

\section{WHAT TO DO WITH ABNORMAL STRESS-FIRST STUDIES?}

Mathur et al fill in another gap in the stressonly literature, namely what happens to the abnormal 
stress-first patients? The majority of patients with abnormal images (63\%) returned for additional rest images. Interestingly, they demonstrate that the other $37 \%$ could be managed clinically without additional rest imaging. Compared to those patients with abnormal stress-rest images, the abnormal stress-only patients had a larger summed stress score (5.4 vs $4.6, P=.03$ ), underwent revascularization more often $(P<.05)$, but had a similar annualized adverse cardiac event rate $(0.9$ vs $0.4 \%, P=.6$ ). The purpose of rest imaging is to characterize the quality of the stress perfusion defects (reversible vs non-reversible). However, an absolute need for such a distinction may be overemphasized. In patients who have undergone previous testing, performing the rest images may be unnecessary as it is unlikely that a previously fixed defect on a full rest-stress study would become reversible (e.g. in the presence of $\mathrm{Q}$ waves on rest ECG, visible thinning of the myocardium, and absent wall motion). ${ }^{18}$ Furthermore, trivial stress perfusion defects in patients with other significant comorbidities, advanced age, or with no indication for coronary revascularization can be safely managed medically.

In the past 30 years, we have transitioned from planar imaging to SPECT imaging, from Tl-201 imaging to Tc-99m imaging, from oral dipyridamole to intravenous regadenoson. Now is the time to transition from the rigid, one-size-fits all rest-stress $/ 10-30 \mathrm{mCi}$ protocol to patient centered, radiation saving, individualized, and efficient protocols which do not sacrifice quality or diagnostic and prognostic utility of the modality. ${ }^{19}$

\section{References}

1. Mathur S, Heller GV, Bateman TM, Ruffin R, Yekta A, Katten D et al. Clinical value of stress-only Tc-99m SPECT imaging: Importance of attenuation correction. J Nucl Cardiol 2012. doi: 10.1007/s12350-012-9633-7.

2. Shaw LJ, Hage FG, Berman DS, Hachamovitch R, Iskandrian A. Prognosis in the era of comparative effectiveness research: Where is nuclear cardiology now and where should it be? J Nucl Cardiol 2012;19:1026-43.

3. Gibson PB, Demus D, Noto R, Hudson W, Johnson LL. Low event rate for stress-only perfusion imaging in patients evaluated for chest pain. J Am Coll Cardiol 2002;39:999-1004.

4. Chang SM, Nabi F, Xu J, Raza U, Mahmarian JJ. Normal stressonly versus standard stress/rest myocardial perfusion imaging: Similar patient mortality with reduced radiation exposure. J Am Coll Cardiol 2010;55:221-30.
5. Duvall WL, Wijetunga MN, Klein TM, Razzouk L, Godbold J, Croft LB, et al. The prognosis of a normal stress-only Tc-99m myocardial perfusion imaging study. J Nucl Cardiol 2010;17: 370-7.

6. Duvall WL, Wijetunga MN, Klein TM, Hingorani R, Bewley B, Khan SM, et al. Stress-only Tc-99m myocardial perfusion imaging in an emergency department chest pain unit. J Emerg Med 2012;42:642-50.

7. Gemignani AS, Muhlebach SG, Abbott BG, Roye GD, Harrington DT, Arrighi JA. Stress-only or stress/rest myocardial perfusion imaging in patients undergoing evaluation for bariatric surgery. J Nucl Cardiol 2011;18:886-92.

8. Hesse B, Tagil K, Cuocolo A, Anagnostopoulos C, Bardies M, Bax J, et al. EANM/ESC procedural guidelines for myocardial perfusion imaging in nuclear cardiology. Eur J Nucl Med Mol Imaging 2005;32:855-97.

9. Gopal A, Nasir K, Ahmadi N, Gul K, Tiano J, Flores M, et al. Cardiac computed tomographic angiography in an outpatient setting: An analysis of clinical outcomes over a 40 -month period. J Cardiovasc Comput Tomogr 2009;3:90-5.

10. Duvall WL, Baber U, Levine EJ, Croft LB, Henzlova MJ. A model for the prediction of a successful stress-first Tc-99m SPECT MPI. J Nucl Cardiol 2012. doi:10.1007/s12350-012-9606-x.

11. Heo J, Kegel J, Iskandrian AS, Cave V, Iskandrian BB. Comparison of same-day protocols using technetium-99m-sestamibi myocardial imaging. J Nucl Med 1992;33:186-91.

12. Depuey EG, Ata P, Wray R, Friedman M. Very low-activity stress/ high-activity rest, single-day myocardial perfusion SPECT with a conventional sodium iodide camera and wide beam reconstruction processing. J Nucl Cardiol 2012;19:931-44.

13. Herzog BA, Buechel RR, Katz R, Brueckner M, Husmann L, Burger IA, et al. Nuclear myocardial perfusion imaging with a cadmium-zinc-telluride detector technique: Optimized protocol for scan time reduction. J Nucl Med 2010;51:46-51.

14. Levin DC, Rao VM, Parker L, Frangos AJ. The sharp reductions in medicare payments for noninvasive diagnostic imaging in recent years: Will they satisfy the federal policymakers? J Am Coll Radiol 2012;9:643-7.

15. Heller GV, Bateman TM, Johnson LL, Cullom SJ, Case JA, Galt $\mathrm{JR}$, et al. Clinical value of attenuation correction in stress-only Tc99m sestamibi SPECT imaging. J Nucl Cardiol 2004;11:273-81.

16. Thompson RC, Heller GV, Johnson LL, Case JA, Cullom SJ, Garcia EV, et al. Value of attenuation correction on ECG-gated SPECT myocardial perfusion imaging related to body mass index. J Nucl Cardiol 2005;12:195-202.

17. Ceylan Gunay E, Erdogan A, Yalcin H, Ozcan Kara P. Prone imaging allows efficient radiopharmaceutical usage by obviating the necessity of a rest study in Tc-99m-methoxyisobutylisonitrile myocardial perfusion scintigraphy. Nucl Med Commun 2011;32: 284-8.

18. Ryan JJ, Mehta R, Thiruvoipati T, Ward RP, Williams KA. Stressonly imaging in patients with prior SPECT MPI: A simulation study. J Nucl Cardiol 2012;19:311-8.

19. Depuey EG, Mahmarian JJ, Miller TD, Einstein AJ, Hansen CL, Holly TA, et al. Patient-centered imaging. J Nucl Cardiol 2012; 19:185-215. 